Notfall Rettungsmed $2015 \cdot 18: 267$

DOI 10.1007/s10049-015-0030-z

Online publiziert: 27. Mai 2015

(c) Springer-Verlag Berlin Heidelberg 2015

\section{J. Hinkelbein ${ }^{1} \cdot$ H. Moecke ${ }^{2}$}

${ }^{1}$ Klinik für Anästhesiologie und Operative Intensivmedizin, Universitätsklinikum Köln (AöR), Köln, Deutschland

${ }^{2}$ Asklepios Institut für Notfallmedizin, c/o Asklepios Klinik St.Georg Hamburg, Hamburg, Deutschland

\title{
Notfallmedizin unter Extrembedingungen
}

Liebe Leserinnen und Leser,

in diesem Heft der Notfall + Rettungsmedizin mit dem Leitthema „Notfallmedizin unter Extrembedingungen " möchten wir Ihnen verschiedene, breit gefächerte Themen aus der Notfallmedizin präsentieren, mit denen man nicht alle Tage in Berührung kommt, die andererseits aber in diversen Spezialbereichen große Relevanz besitzen.

Hatten Sie bereits mit dem notfallmedizinischen MANV-Szenario einer Bombenexplosion zu tun? Oder hatten Sie auf einem Ihrer letzten Transatlantikflüge einen medizinischen Notfall an Bord eines Luftfahrzeugs, weit ab jeglicher medizinischer Hilfe? Im Weltall werden Sie wohl in der nächsten Zeit nicht sein, aber auch dort existieren notfallmedizinische Konzepte, ja sozusagen auch Routinekonzepte; denn auch Astronauten an Bord einer Raumstation können krank oder verletzt werden.

Als gutes Beispiel für Spezialbereiche eignen sich Notfälle im Weltall, z. B. auf der Internationalen Raumstation, ISS, oder zukünftig auch auf länger andauernden Missionen zum Mars. Hilfe von Außen ist im Falle eines Notfalls sicher nicht zu erwarten, weswegen auch auf keinerlei materielle und personelle Hilfe von außerhalb zurückgegriffen werden kann. Man ist sozusagen ,auf sich selbst gestellt". Entsprechend wichtig ist adäquate notfallmedizinische Ausbildung der Besatzung, aber auch das richtige Material, Konzepte und SOPs müssen vorhanden sein. Hierzu gibt die Arbeit von Komorowski et al. eine sehr interessante Übersicht. Auch das Team muss entsprechend trainiert sein und suffizient interagieren:
Stichwort CRM - Crew Ressource Management.

Wie oft kam es schon zu einer Reanimationssituation im Weltraum? Noch nie! Können wir nach ROSC Astronauten überhaupt lebend zurück zur Erde bringen? Wahrscheinlich nicht. Also brauchen wir hierzu gar kein Konzept? Doch, wir brauchen es! Allein schon aus ethischer Sicht müssen Strukturen vorhanden sein, um auch mit diesen seltenen, außergewöhnlichen Notfällen zurechtzukommen. Auch auf diesem sehr schmalen, exotischen Feld sind international deutliche Forschungsbemühungen an einigen wenigen Zentren vorhanden, wie der Beitrag von Braunecker et al. zeigt.

Man wird nun vielleicht denken, „Was geht mich der Weltraum an?" Derartige Konzepte sind aber, zumindest in Ansätzen, auch auf unserer Erde notwendig. Denken Sie beispielsweise an Hochsee-Bohrinseln oder vielleicht auch nur an eine ländliche Rettungswache, weit weg von der nächsten großen Stadt, wenn es dunkel ist und kein Backup oder Hubschrauber zur Verfügung steht. Aber auch im Linienflugbetrieb kann es nahezu regelhaft derartige medizinische Probleme geben, wie der Artikel von Neuhaus et al. zeigt.

Neben der notfallmedizinischen Qualifikation bzw. Kompetenz, die für die Bewältigung notwendig ist, sind $\mathrm{u}$. U. weitere körperliche Leistungsmerkmale erforderlich. Diese müssen oftmals unter simulierten Spezialsituationen in kritischen Umgebungen aufwändig trainiert werden (z. B. Windenrettung oder Wasserrettung).

Auch die Sicherheit des Rettungsteams spielt bei der Versorgung eine essenzielle Rolle. „Safety first“ gilt universell, im
Weltraum, in der Luftfahrt oder auch auf dem Boden. Diesen Aspekt zeigen Peleg et al. auf, die einen sehr interessanten Beitrag über die notfallmedizinischen Auswirkungen von terroristischen Bombenanschlägen in Israel präsentieren. Hier ist nicht nur, wie man vielleicht fälschlich glaubt, der primäre Anschlag von Relevanz, denn oftmals wird auch gezielt auf die Rettungsteams ein weiterer Anschlag verübt, sog. „second attack“. Auch hier$\mathrm{zu}$ sind Spezialkonzepte unerlässlich und man kann aus den bisherigen Erfahrungen lernen.

Derartige Extremsituationen, von denen wir nur einen Auszug präsentieren können, sind häufig nicht mit den Standardelementen aus Prozess- und Strukturqualität der regelhaften präklinischen Notfallmedizin zu bewältigen. Mehr noch, all diese Aspekte verschiedener Extrembereiche erfordern oftmals Spezialkonzepte, von denen man aber auch für die tägliche Routine beim bodengebundenen Rettungsdienst/Notarztdienst wertvolle Impulse ableiten kann - nein - ableiten muss. Es ist daher nicht nur interessant, darüber zu lesen, wir müssen auch davon lernen - für eine stetig besser werdende Notfallmedizin.

Wir wünschen Ihnen viel Spaß bei dieser Zusammenstellung diverser notfallmedizinischer Themenkomplexe aus Extrembereichen.

\section{Korrespondenzadresse}

\section{Priv.-Doz. Dr. J. Hinkelbein}

Klinik für Anästhesiologie und Operative Intensivmedizin

Universitätsklinikum Köln (AöR)

Kerpener Straße 62, 50937 Köln

jochen.hinkelbein@uk-koeln.de 